\title{
The Effects of Aerobic Versus Resistance Training on Cardiovascular Fitness in Obese Sedentary Females
}

\author{
Sarika Chaudhary*, MSPT, Manpreet Kaur Kang, MSPT; Jaspal Singh Sandhu, MS, FAIS, FASM
}

\section{Authors' Affiliation: \\ Faculty of Sports Medicine and Physiotherapy, Guru Nanak Dev University, Amritsar, Punjab, India

\footnotetext{
* Corresponding Author; Address: Faculty of Sports Medicine and Physiotherapy, Guru Nanak Dev University, Amritsar, Punjab, India

E-mail: sarika5april@rediffmail.com
}

Received: May 17, 2010

Accepted: Nov 01, 2010

Key Words: Aerobic exercise; Resistance training; Obesity; Heart rate; Physical fitness

\section{Abstract}

Purpose: The present study was designed to evaluate the effects of aerobic and strength training on cardiac variables such as blood pressure, heart rate (HR), and metabolic parameters like cholesterol, high density lipoprotein (HDL), triglycerides and anthropometric parameters of obese women of Punjab.

Methods: This study was performed as an experimental study, in which subjects were randomly selected. There were thirty obese women, aged between 35-45yrs with body mass index (BMI) of above 30. Subjects were grouped into control $(n=10)$, aerobic training $(n=10)$ and resistance training $(n=10)$. Aerobic training was given for three days a week at $60-70 \%$ of maximum HR for 6 weeks. Resistance training (Delorme and Watkins Technique) was given for alternate days for 6 weeks. HR and blood pressure were measured before and after the exercise. Recovery HR was also measured.

Results: The findings of the study indicate statistically significant differences in recovery heart rate [Pre-exercise: $97.40 \pm 5.378$ (mean \pm standard deviation (SD)), post-exercise: $90.70 \pm 4.599, t=8.066$, $P<0.001$ ] and in post-diastolic blood pressure [Pre-exercise: 85 \pm 3.265 , post-exercise: $86.20 \pm 2.820, P<0.001]$ in aerobic training and in systolic blood pressure [Pre- and post-exercise] in both training groups $(P<0.001)$. Significant differences were observed in very low-density lipoprotein [pre-exercise: $28.10 \pm 1.415$, postexercise: $26.86 \pm 0.760, t=5.378$ ] and HDL [pre-exercise: $45.40 \pm 3.533$, post-exercise: $53.60 \pm 3.134, t=6.318$ ] levels in aerobic training group with $P<0.001$. BMI and body fat percentage showed significant improvements in both training groups.

Conclusion: Aerobic training is more beneficial and can be used as a preventive measure in patients who are at risk of developing cardiovascular diseases due to obesity. 


\section{INTRODUCTION}

A widespread concern exists about the low level of vigorous physical activity and high rates of sedentary behavior in women. The lack of physical activity in daily life induces obesity and increases the risk of diseases such as diabetes mellitus, hypertension and heart diseases. Obesity and physical inactivity comprise an important worldwide epidemic that has been linked to the metabolic syndrome ${ }^{[1]}$. Body mass index (BMI) appears to be the best index of obesity, as it approximates adiposity and fat distribution in adults $^{[2]}$. There is an increasing evidence shows that the associations between BMI, percentage of body fat and body fat distribution differ across populations ${ }^{[3]}$.

According to WHO estimation in 2005, 400 million adults $(9.8 \%)$ are obese, with higher rates of obesity among women than men. Increased weight gain, which is associated with increased lean and fat mass, along with the associated increase in total blood volume maybe accompanied by an increase in stroke volume, cardiac output and circulatory preload and afterload that can lead to left ventricular hypertrophy and sustained rise in blood pressure ${ }^{[4]}$.

Regular exercise can markedly reduce body weight and fat mass without dietary caloric restriction in overweight individuals. A minimum of $60 \mathrm{~min}$, but most likely 80-90 min of moderate-intensity physical activity per day maybe needed to avoid or limit weight regain and to prevent and treat cardiovascular diseases in formerly overweight or obese individuals ${ }^{[5]}$.

Weight reduction in obesity brought about by physical training has been associated with numerous metabolic adaptations including preservation of lean body mass (LBM), improved muscle endurance, increased insulin sensitivity, improved high-density lipoprotein cholesterol, low-density lipoprotein cholesterol ratio and improved ability of the muscle cell to take up glucose and metabolize fat ${ }^{[6]}$.

Moderate to high intensity aerobic training results in an improvement in the blood lipid profile $\mathrm{e}^{[7]}$. The most recent studies in patients with established heart disease suggest that a relatively high intensive, yet aerobic exercise training improves the intrinsic pump capacity of the myocardium ${ }^{[8]}$. Heart rate recovery (HRR) or the rate at which heart rate (HR) declines from either maximal or sub-maximal exercise to resting levels is identified as a powerful and independent predictor of cardiovascular and all-cause mortality in healthy adults ${ }^{[9]}$. Active recovery aids in lactate removal following intense exercise and reducing performance decrements in subsequent bout. The muscles responsible for initial increases in lactate concentration during intense exercise require a recovery period in excess of three minutes to modify intracellular lactate metabolism resulting in lactate gradient in favor of uptake by the same muscle ${ }^{[10]}$.

The constant stress of aerobic exercise forces the heart to gradually enlarge. Thus, future exercise eventually requires less effort. Heart rate increases acutely immediately following a work bout and is affected by the amount of resistance, the number of repetitions and the muscle mass involved in the contraction small vs. large mass exercises ${ }^{[11]}$. A previous study showed that weight loss improves HRR in overweight and obese men, suggesting that HRR maybe a modifiable risk factor via changes in body weight $^{[12]}$.

Strength training with greater intensity (fewer repetitions and greater weight) with multiple sets can elicit greater improvements in strength and power, and may not be appropriate for older nonathletic participants $^{[13]}$.

In terms of chronic adaptations, there appears to be a reduction in heart rate following resistance training, which is considered beneficial ${ }^{[11]}$. Aerobic and resistance trainings induce improvement in body fat composition and have favorable metabolic effects in obese women with severe eating disorders ${ }^{[14]}$. The present study was designed to obtain data on the obese females of Punjab. The reason is that although most of Punjab obese females stick to sedentary lifestyle and high calorie food habits and are not aware of the hazardous effects of obesity and sedentary lifestyle, there are not many related studies that have been carried out in this population.

The main objective of the present study was to assess the efficacy of aerobic versus resistance training in improving cardiovascular parameters (i.e HR, blood pressure), anthropometric parameters (such as BMI, body fat percentage) and blood lipid profile (such as high density lipoprotein (HDL), cholesterol and 
triglycerides) in obese women. The hypothesis of the study was that aerobic exercise may prove to be more beneficial than resistance training in improving cardiovascular fitness of obese women.

\section{METHODS AND SUBJECTS}

This study was performed as an experimental study, in which subjects were randomly selected. There were thirty obese women, aged 35-45 years, recruited from Amritsar, Punjab (India). Only healthy subjects without any history of pathologic or orthopedic limitation were included in the study. No subject was currently engaged in any of the other exercise programs. They were familiarized with the training program and were informed about the possible risks and benefits involved with the study both verbally and in writing before obtaining written informed consent. Subjects with a body mass index of above 30 were chosen and instructed not to take fried products and foods with high sugar content.

\section{Subjects:}

The study protocol was approved by the institutional ethics review board, Guru Nanak Dev University. Subjects were grouped into control $(\mathrm{n}=10)$, aerobic training $(\mathrm{n}=10)$ and resistance training $(\mathrm{n}=10)$.

\section{Apparatus used:}

Sphygmomanometer, stethoscope, weighing machine, kinanthropometric rod, treadmill (Viva Fitness T 602), weight cuffs, polar heart rate monitor (S410 with T61 transmitter and 410 wrist receiver), automated analyzer, centrifuge machine and body composition measurement.

Height was measured using kinanthropometric rod. Weight was measured by the weighing machine. BMI was calculated as weight $(\mathrm{kg}) /$ height $(\mathrm{m} 2)$ and body fat percentage was calculated by using formula as body fat $\%=1.37 \times$ BMI -3.47 (Durnin \& Womersley, 1974).

\section{Exercise groups:}

All participants were familiar with the exercise testing protocol by going through preliminary exercise test. Polar heart rate monitor (S 4 10) was used to monitor the heart rate continuously. In the polar heart rate monitor, elastic strap was secured around the subject's chest. The receiver was worn in the subject's wrist and the monitor was activated to measure the heart rate. Systolic (SBP) and diastolic blood pressure (DBP) were measured in a sitting position on the upper arm using an inflatable cuff and mercury sphygmomanometer before and after the exercise.

\section{Training program:}

Aerobic Training: Stretching and low intensity exercises of 10 minutes were given in each training session as warm-up prior to training. The aerobic training was given for three alternate days in a week at $60-70 \%$ of maximum HR for 6 weeks, up to the exhaustion level of the subjects. Polar heart rate monitor was used continuously to maintain $60 \%$ to $70 \%$ of maximum HR during the aerobic exercise. Heart rate, systolic and diastolic blood pressure were measured before and after the exercise in the sitting position. Recovery heart rate was measured at third minute of post exercise session in the aerobic training program. After the training session, cool down was given for 5-10 minutes.

Maximum heart rate was calculated by using the formula:

$$
\mathrm{HR}_{\text {max }}=220 \text {-age }
$$

Resistance training: Resistance training program was also given for alternate days for 6 weeks. In resistance training four sets and 10 repetitions were performed by the subjects, based on the Delorme and Watkins technique. It was started with 10 lifts with $50 \%$ of 10 $\mathrm{RM}$, then $75 \%$ of $10 \mathrm{RM}$ and progressed to $100 \%$ of 10 RM. Seven different types of exercises were abdominal curl ups, biceps curls, triceps extension, back extension, leg curls, side leg raises and knee extension. The control group did not perform any type of training but was given a diet chart to follow.

\section{Blood Sample Analysis:}

Blood sample was drawn from the antecubital vein. Subjects were on 12 hours fasting prior to test and were instructed not to take fried products and foods with high sugar content during the 6 weeks of intervention period and were required to follow their "usual" diet. All blood samples were analyzed by automated analyzer (Minitecno I.S.E group) and were obtained in early morning. Blood samples for lipid analysis $(10 \mathrm{ml})$ were drawn only at baseline (fasting). After the sample 
was drawn, it was centrifuged at $3000 \mathrm{rpm}$ (Eltek, TC $4815 \mathrm{D})$ for $5 \mathrm{~min}$. Subsequently, the serum was drawn off and placed in a small tube and then was kept at 4 degrees Celsius. Serum cholesterol and triglyceride concentrations were measured with an automated analyzer using standard enzymatic techniques. HDL was measured after removal of very low density lipoprotein (VLDL) and low density lipids (LDL) from samples. Lipid profile testing was done before starting the training program at the first week and then after the six weeks of training by making the subjects sit on the chair.

\section{Statistical Analysis:}

All data are presented as mean \pm standard deviation (SD). The pre and post-test data were analyzed with a statistical paired sample t-test. Statistical significance was accepted at $P<0.05$. An alpha level of 0.001 was used in determining statistical significance using the SPSS program for Windows, version 14.0.

\section{RESULTS}

The pre to post training results of paired t-test for maximum heart rate showed a statistically significant difference in aerobic training [pre-exercise: $109.40 \pm 2.988 \quad$ (mean \pm SD), post-exercise: $116.70 \pm 2.626, \mathrm{t}=7.643, P<0.001]$ in comparison with resistance training [pre-exercise: $82.20 \pm 5.432$, postexercise: $80.90 \pm 4.306, \mathrm{t}=2.327, P=0.04]$. The $3^{\text {rd }}$ min heart rate recovery results of paired t-test in case of aerobic training showed significant changes $(t=8.066$, $P<0.001)$ as compared to resistance training $(\mathrm{t}=2.121$, $P=0.06)$. Results of paired t-test in aerobic training in pre SBP week 1 to week $6(\mathrm{t}=4.114, P=0.003)$ and post SBP week 1 to week $6(\mathrm{t}=7.108, P<0.001$ showed more significant changes than resistance training in pre SBP wk 1 to wk $6(\mathrm{t}=4.617, P<0.001)$ and post SBP wk 1 to wk $6(\mathrm{t}=4.590, P<0.001)$. Results of paired t-test in aerobic training in case of pre DBP and post DBP showed statistically significant changes $(\mathrm{t}=6.249,7.652$ respectively; $P<0.001)$ as compared to resistance training $(P<0.001)$ and control group $(P=0.4)$. The pre to post training paired t-test of cholesterol, triglycerides, HDL (pre 45.40 \pm 3.533 , post 53.60 $\pm 3.134, \mathrm{t}=6.318$ ) and VLDL (pre 28.10 \pm 1.415 , post $26.86 \pm 0.760, \mathrm{t}=5.378)$ in aerobic training group were significant $(P<0.001)$ as compared to control and resistance training groups. The pre to post training results of weight, BMI and body fat percentage were statistically significant in cases of aerobic and resistance trainings $(P<0.001)$ in comparison with

Table 1: The Pre and post test mean and SD of studied variables in all three groups

\begin{tabular}{|l|cccccc} 
Variables & \multicolumn{2}{|c}{ Aerobic } & \multicolumn{2}{c}{ Resistance } & \multicolumn{2}{c}{ Control } \\
& Pre & Post & Pre & Post & Pre & Post \\
\hline Weight (kg) & $77.30 \pm 5.870$ & $73.40 \pm 6.114$ & $77.10 \pm 6.045$ & $75.40 \pm 5.739$ & $78.00 \pm 5.538$ & $78.70 \pm 4.495$ \\
BMI & $31.93 \pm 1.466$ & $30.32 \pm 1.5383$ & $32.19 \pm 2.571$ & $31.47 \pm 2.362$ & $31.82 \pm 1.503$ & $32.11 \pm 1.378$ \\
Body \% & $40.27 \pm 2.009$ & $38.07 \pm 2.107$ & $40.63 \pm 3.523$ & $39.64 \pm 3.236$ & $40.11 \pm 2.069$ & $40.52 \pm 1.888$ \\
Chol (mg/dl) & $242.70 \pm 21.176$ & $233.00 \pm 19.539$ & $247.50 \pm 13.360$ & $242.60 \pm 13.945$ & $246.70 \pm 15.684$ & $248.30 \pm 16.391$ \\
TG (mg/d) & $140.00 \pm 7.075$ & $134.30 \pm 3.802$ & $140.10 \pm 6.436$ & $136.70 \pm 5.696$ & $143.90 \pm 6.226$ & $145.0 \pm 7.659$ \\
HDL (mg/dl) & $45.40 \pm 3.533$ & $53.60 \pm 3.134$ & $46.10 \pm 5.724$ & $49.40 \pm 4.993$ & $44.80 \pm 4.541$ & $44.50 \pm 5.317$ \\
VLDL (mg/d) & $28.10 \pm 1.415$ & $26.86 \pm 0.760$ & $27.82 \pm 27.34$ & $1.48 \pm 1.139$ & $28.78 \pm 1.245$ & $29.00 \pm 1.531$ \\
Pre SBP (mm Hg) & $128.10 \pm 4.954$ & $124.20 \pm 2.820$ & $129.70 \pm 4.498$ & $126.70 \pm 3.713$ & $125.80 \pm 4.263$ & $125.80 \pm 4.263$ \\
Post SBP (mm Hg) & $131.70 \pm 4.083$ & $123.70 \pm 2.540$ & $131.70 \pm 4.667$ & $127.20 \pm 3.910$ & $126.40 \pm 4.402$ & $126.40 \pm 4.402$ \\
Pre DBP (mm Hg) & $85.00 \pm 3.265$ & $81.80 \pm 3.119$ & $83.70 \pm 2.311$ & $83.50 \pm 2.877$ & $84.80 \pm 4.131$ & $84.80 \pm 4.131$ \\
Post DBP (mm Hg) & $86.20 \pm 2.820$ & $80.70 \pm 2.750$ & $83.00 \pm 2.160$ & $82.60 \pm 1.349$ & $85.10 \pm 4.012$ & $85.10 \pm 4.012$
\end{tabular}

BMI: Body Mass Index; Chol: Cholesterol; Trig-Triglycerides; HDL: High Density Lipids; VLDL: Very Low Density Lipids; SBP: Systolic Blood Pressure; DBP: Diastolic Blood Pressure 
Table 2: The t-values and 95\% confidence intervals of variables in all three groups

\begin{tabular}{|c|c|c|c|c|c|c|c|c|c|}
\hline \multirow{3}{*}{ Variables } & \multicolumn{3}{|c|}{ Aerobic } & \multicolumn{3}{|c|}{ Resistance } & \multicolumn{3}{|c|}{ Control } \\
\hline & \multirow[t]{2}{*}{ t value } & \multicolumn{2}{|c|}{$95 \% \mathrm{CI}$} & \multirow[t]{2}{*}{ t value } & \multicolumn{2}{|c|}{$95 \% \mathrm{CI}$} & \multirow[t]{2}{*}{ t value } & \multicolumn{2}{|c|}{$95 \%$ CI } \\
\hline & & Upper & Lower & & Upper & Lower & & Upper & Lower \\
\hline Weight (kg) & 16.714 & 4.428 & 3.372 & 7.965 & 2.182 & 1.217 & 2.090 & 0.058 & 1.458 \\
\hline BMI & 14.773 & 1.856 & 1.363 & 7.383 & 0.940 & 0.499 & 2.092 & 0.023 & 0.603 \\
\hline Body \% & 14.773 & 2.543 & 1.867 & 7.383 & 1.288 & 0.684 & 2.167 & 0.017 & 0.835 \\
\hline Chol (mg/dl) & 6.746 & 12.952 & 6.447 & 5.770 & 6.820 & 2.979 & 2.667 & 0.242 & 2.957 \\
\hline TG (mg/dl) & 5.378 & 8.807 & 3.592 & 6.278 & 4.625 & 2.174 & 1.009 & 1.365 & 3.565 \\
\hline HDL (mg/dl) & 6.318 & 5.26 & 11.135 & 6.128 & 2.081 & 4.518 & 0.709 & 1.256 & 0.656 \\
\hline VLDL (mg/dl) & 5.378 & 1.761 & 0.178 & 2.092 & 0.998 & 0.038 & 1.009 & 0.273 & 0.713 \\
\hline Pre SBP（mm Hg) & 4.114 & 6.044 & 1.755 & 4.617 & 4.469 & 1.530 & 1.152 & 0.577 & 1.777 \\
\hline Post SBP (mm Hg) & 7.108 & 10.545 & 5.454 & 4.590 & 6.717 & 2.282 & & & \\
\hline Pre DBP (mm Hg) & 6.249 & 4.358 & 2.041 & 0.259 & 1.945 & 1.545 & 0.818 & 0.529 & 1.129 \\
\hline Post DBP (mm Hg) & 7.652 & 7.126 & 3.873 & 0.557 & 2.024 & 1.224 & & & \\
\hline
\end{tabular}

CI: Confidence Interval; BMI: Body Mass Index; Chol: Cholesterol; Trig-Triglycerides; HDL: High Density Lipids; VLDL: Very Low Density Lipids; SBP: Systolic Blood Pressure; DBP: Diastolic Blood Pressure

control group $(P=0.06)$.

\section{DISCUSSION}

The present study evaluated the effects of two different exercise programs without dietary restriction on cardiac fitness variables such as blood pressure and heart rate, metabolic parameters such as cholesterol, HDL, VLDL and triglycerides and anthropometric parameters.
In the present study, aerobic exercise and resistancetraining resulted in reduction in cholesterol and very low density lipid concentrations and improvement in HDL levels while aerobic training showed more statistically significant results than resistance and control group. Aerobic training increases HDL cholesterol. Several studies have confirmed this belief $^{[15]}$ and the results of the present study are in agreement with the above statement. The concentration of HDL is inversely correlated with the risk of coronary heart disease. Low intensity aerobic training may improve the profile of HDL-C and its subfractions in healthy elderly subjects ${ }^{[16]}$. Exercise enhances the

Table 3: The mean, SD, t-value and p-value and 95\% confidence interval of aerobic and resistance groups

\begin{tabular}{|c|c|c|c|c|c|c|}
\hline \multirow{2}{*}{ Variables } & & \multirow{2}{*}{ Mean (SD) } & \multirow{2}{*}{ t value } & \multirow{2}{*}{ P value } & \multicolumn{2}{|c|}{$95 \% \mathrm{CI}$} \\
\hline & & & & & Upper & Lower \\
\hline \multirow{2}{*}{ AEROBIC } & Pre (week 1 HR max) & $109.40(2.988)$ & \multirow{2}{*}{7.643} & \multirow{2}{*}{$<0.001$} & \multirow{2}{*}{9.460} & \multirow{2}{*}{5.139} \\
\hline & Post(week 6 HR max) & $116.70(2.626)$ & & & & \\
\hline \multirow{2}{*}{ RESISTANCE } & Pre (week 1 HR max) & $82.20(5.432)$ & \multirow{2}{*}{2.327} & \multirow{2}{*}{0.04} & \multirow{2}{*}{2.564} & \multirow{2}{*}{0.035} \\
\hline & Post(week 6 HR max) & $80.90(4.306)$ & & & & \\
\hline
\end{tabular}

CI: Confidence Interval; HR max: Heart Rate Maximum (beats/min) 
Table 4: The mean, SD, t-value and p-value of aerobic and resistance groups

\begin{tabular}{|c|c|c|c|c|c|c|}
\hline \multirow{2}{*}{ Variables } & & \multirow{2}{*}{ Mean (SD) } & \multirow{2}{*}{ t value } & \multirow{2}{*}{ P value } & \multicolumn{2}{|c|}{$95 \%$ CI } \\
\hline & & & & & Upper & Lower \\
\hline \multirow{2}{*}{ AEROBIC } & Pre (week 1 rHR $3 \mathrm{~min}$ ) & $97.40(5.378)$ & \multirow{2}{*}{8.066} & \multirow{2}{*}{$<0.001$} & \multirow{2}{*}{8.579} & \multirow{2}{*}{4.820} \\
\hline & Post (week 6 rHR 3min) & $90.70(4.599)$ & & & & \\
\hline \multirow{2}{*}{ RESISTANCE } & Pre (week 1 rHR 3min) & $80.40(5.758)$ & \multirow{2}{*}{2.121} & \multirow{2}{*}{0.06} & \multirow{2}{*}{2.066} & \multirow{2}{*}{0.066} \\
\hline & Post (week 6 rHR 3min) & $79.40(4.595)$ & & & & \\
\hline
\end{tabular}

production of the enzymes that facilitate HDL transportation of cholesterol back into the liver to be broken down. Thus, HDL acts as a scavenger in the reverse transport of cholesterol.

In the present study, triglyceride concentration also showed a statistically significant reduction in aerobic training group. Aerobic training reduces triglyceride concentration $^{[17]}$ and the findings of the present study support the aforementioned statement. The greater utilization of free fatty acid in the trained state is fueled by increased lipolysis of muscle triglyceride. Epidemiological research has decisively demonstrated that low concentrations of total cholesterol and lowdensity lipoprotein cholesterol (LDL-C) and high levels of high-density lipoprotein cholesterol (HDL-C) are associated with decreased coronary heart disease ${ }^{[11]}$

Although the present study did not include a diet modification component in the two training groups, body weight, body mass index and body fat percentage showed a marked reduction when observed individually in both groups. The alterations in body composition are most often attributed to aerobic exercise with a decrease in fat weight and a maintenance or slight increase in fat-free mass indicating the importance of aerobic exercise in burning calories and losing body fat ${ }^{[11]}$. Thus, the results of the present study are in agreement with the above statement. Several studies suggested that exercise alone has subtle effects on body weight in overweight subjects, training thus mobilize fat ${ }^{[15]}$. Increased weight gain which is associated with increase in both lean and fat mass, along with the associated increase in total blood volume can be accompanied by an increase in stroke volume, cardiac output, circulatory preload and afterload and can lead to left ventricular hypertrophy and sustained rise in blood pressure $^{[4]}$.

Reduction in the recovery heart rate has been observed in the present study. Statistically significant changes have been observed in recovery heart rate in case of aerobic training. The decrease in the heart rate which has been observed in the present study can be justified by the fact that exercise training creates an imbalance between the tonic activity of sympathetic accelerator and parasympathetic depressor neurons in favor of greater vagal dominance, a response mediated primarily by increased parasympathetic activity and a small decrease in sympathetic discharge ${ }^{[18]}$. The results of the present study could be due to the fact that active recovery immediately after the event encourages recovery and reduces muscle lactate levels faster than complete rest and enhances performance, as there is continued use of free fatty acids as fuel during active recovery.

Regular training confers beneficial effects on the heart as well the entire body. This occurs partly because exercise training improves skeletal muscle work capacity and reduces resistance, thus increases conductance in the peripheral circulation. Extrinsic modulation of the heart alters and improves the intrinsic pump capacity of the heart ${ }^{[8]}$.

In the present study, the decrease in both systolic and diastolic blood pressure was observed after aerobic and strength training. Statistically significant differences were observed more frequently in aerobic program, maybe because of favorable changes in vascular compliance, which might have occurred after exercise training thus could reduce peripheral resistance. The 
blood pressure during aerobic exercise is much lower than the response to heavy resistance exercise ${ }^{[19]}$ and the results of the present study are in accordance with the above statement. The mechanisms by which aerobic exercise may lower blood pressure partly involve with the effects of two hormones, epinephrine and nor-epinephrine. Indeed, aerobic exercise can reduce the blood level of nor-epinephrine which can limit the vasoconstriction of the arterioles and decrease the peripheral resistance to blood pressure. Simultaneously, a slight reduction in sympathetic neural activity may help to modify this decrease in blood pressure $^{[11]}$.

HR acutely elevates immediately after a work bout and is affected by the amount of resistance, the number of repetitions and muscle mass involved in the contraction (small vs. large mass exercises) ${ }^{[11]}$. During a resistance exercise bout, systolic and diastolic blood pressures may show dramatic increases, which suggest that patients with cardiovascular diseases should be monitored more attentively ${ }^{[20]}$. The effects of resistance training on blood pressure are varied due largely to differences in the study design.

This study is restricted to obese females aged from 35 to 45 years. Future studies can be performed on obese males. Moreover, very obese females with BMI $\geq 40$ were not included in this study.

\section{CONCLUSION}

The results of the present study indicate that aerobic training is more beneficial than resistance training in improving cardiovascular fitness and can be used as a preventive measure in patients who are at risk of developing cardiovascular diseases due to obesity and also to prevent from lifestyle related diseases.

Aerobic training increases energy expenditure by activation of lipolysis and affects the reduction of body weight and body fat percentage.

\section{ACKNOWLEDGMENTS}

Gratitude is expressed to the subjects who participated in this study as well as to each of the assistants who were instrumental in the collection of the data. The researchers independently collected, analyzed, and interpreted the results and have no financial interests in the results of this study. The study protocol was approved by the Institutional Ethics Review Board, Guru Nanak Dev University

Conflict of interests: None

\section{REFERENCES}

1. Ferrari CKB. Metabolic syndrome and obesity: Epidemiology and Prevention by physical activity and exercise. $J$ Exerc Sci Fit. 2008;62:87-96.

2. Dimkpa U, Andrew C. Independent multiple correlates of post-exercise systolic blood pressure recovery in healthy adults. Int J Exerc Sci. 2010;31:25-35.

3. Appropriate body-mass index for Asian populations and its implications for policy and intervention strategies Lancet WHO expert consultation Public Health. 2004;363:157-63.

4. Peter G. Kopelman Obesity as a medical problem. Nature. 2000; Pp:404.

5. Lakka TA, Bouchard C. Physical activity, obesity and cardiovascular diseases. Handb Exp Pharmacol. 2005; 170:137-63.

6. Thomas DP, Belko AZ, Mulroy GL, et al. combined effects of exercise and restriction of energy intake on moderately obese Women. Br J Sports Med. 1986;20:84-8. 
7. Leon AS, Sanchez OA. Response of blood lipids to exercise training alone or combined with dietary intervention. $J$ Am College of Sports Med. 2001;33:S502-515.

8. Kemi, OJ, Wisloff U. High-intensity aerobic exercise training improves the heart in health and disease. J Cardiopulm Rehabil Prev. 2010;30:2-11.

9. Dimkpa U Post-Exercise Heart Rate Recovery: An index of cardiovascular fitness. J Exe Physiol. 2009;12:19-22.

10. Declan A.J Connolly, Kevin M.Brennan and Christie D.Lauzon Effects of Active versus Passive Recovery on power output during repeated bouts of short term, high intensity exercise. J Sports Sci Med. 2003;2:47-51

11. Kravitz L. Resistance Training: Adaptations and Health Implications. Idea Today Health Publications. 1996;14:3846.

12. Brinkworth G, Noakes M, Buckley J, Clifton P. Weight loss improves heart rate recovery in overweight and obese men with features of the metabolic syndrome. Am Heart J. 2006;152(4):693.

13. Pollock ML, Vincet KR, Corbin C, Pangrazi B. Resistance Training for Health. The President's Council on Physical Fitness and Sports Research Digest. 1996; Series2.

14. Fenkci S, Sarsan A, Rota S, Ardic F. Effects of resistance or aerobic exercises on metabolic parameters in obese women who are not on a diet. Advances in Therapy. 2008;23:404-13.

15. Banz WJ, Maher MA, Thompson WG, et al. Effects of resistance versus aerobic training on coronary artery disease risk factors. Exp Biol Med. 2003;228:434-440.

16. Sunami Y, Motoyama M, Kinoshita F, et al. Effects of low-intensity aerobic training on the high-density lipoprotein cholesterol concentration in healthy elderly subjects. Metabolism. 1999;48:984-8.

17. Kelley GA, Kelley KS, Tran ZV. Aerobic exercise, lipids and lipoproteins in overweight and obese adults: a meta analysis of randomized controlled trials. Int J Obes. 2005;29:881-93.

18. Mcardle WD, Katch FI, Katch VL. Exercise physiology: Energy nutrition and Human Performance. $5^{\text {th }}$ ed. Lippincott Williams \& Wilkins; Baltimore. 2001; P:140.

19. Kravitz L Exercise and resting blood pressure. Health Publications. 2001;16:21-22.

20. Kelley GA, Kelley KS. Progressive resistance exercise and resting blood pressure. Hypertension. 2000;35:838. 